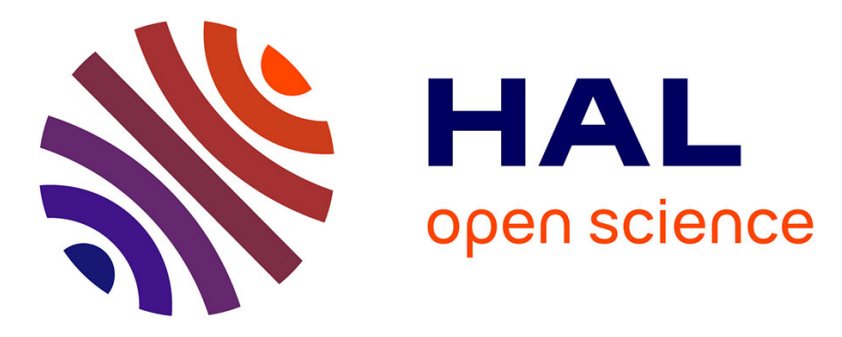

\title{
Random Phase Infinite Coherent States: Construction and Dynamics
}

\author{
Alain Joye, Marco Merkli
}

\section{To cite this version:}

Alain Joye, Marco Merkli. Random Phase Infinite Coherent States: Construction and Dynamics. Federico Bonetto, David Borthwick, Evans Harrell and Michael Loss. Mathematical Problems in Quantum Physics, 717, American Mathematical Society, 2018, Contemporary Mathematics, 978-14704-3681-0. hal-01990458

\section{HAL Id: hal-01990458 https://hal.science/hal-01990458}

Submitted on 1 Feb 2019

HAL is a multi-disciplinary open access archive for the deposit and dissemination of scientific research documents, whether they are published or not. The documents may come from teaching and research institutions in France or abroad, or from public or private research centers.
L'archive ouverte pluridisciplinaire HAL, est destinée au dépôt et à la diffusion de documents scientifiques de niveau recherche, publiés ou non, émanant des établissements d'enseignement et de recherche français ou étrangers, des laboratoires publics ou privés. 


\title{
Random Phase Infinite Coherent States: Construction and Dynamics
}

\author{
Alain Joye and Marco Merkli \\ Abstract. We consider an infinitely extended reservoir of Boson coherent \\ states characterized by a given spatial density of modes and i.i.d. random \\ phases. We construct its Hilbert space representation which has a random \\ part and is expressed by means of Ito stochastic integrals. We study the open \\ system dynamics of an $N$-level system coupled to the random infinite coherent \\ state by an energy conserving interaction. We show that the coherent state \\ reservoir induces faster system decoherence than a thermal reservoir.
}

\section{Introduction}

Coherent states have played an important role in quantum mechanics since the early days of the theory. Schrödinger discussed them as early as 1926 and noted that they behave in many respects like classical states $[\mathbf{2 0}]$. Later on, thanks to the work of Glauber $[\mathbf{7}, \mathbf{8}]$, it was realized that these states are particularly suited to describe optical coherence, which gave them their name. In particular, the electromagnetic radiation generated by a classical current is a multimode coherent state, and so is the light produced by a laser in certain regimes $[\mathbf{1 3}, \mathbf{1 4}]$. Therefore, coherent states are building blocks of modern quantum optics $[\mathbf{1 3}, \mathbf{6}, \mathbf{2 1}]$, they lie at the heart of semiclassical analysis, see e.g. $[\mathbf{1 2}, \mathbf{4}]$, and more recently, they found applications in quantum information experiments $[\mathbf{9}]$.

We focus in the current text on the discussion of coherent states with random phases, which is just one aspect of the analysis of [11], where the infinite volume(or thermodynamic) limit of the quantized radiation field in multimode coherent states is considered in more generality.

Consider a free bosonic quantum field confined to a finite box $\Lambda \subset \mathbb{R}^{3}$. As usual, we associate to each discrete mode $k$ a creation and an annihilation operator $a_{k}^{*}$ and $a_{k}$, respectively, satisfying the canonical commutation relations $\left[a_{k}, a_{\ell}^{*}\right]=\delta_{k, \ell}$ (Kronecker symbol). Also, to each $k$ corresponds a family of normalized coherent states $|\alpha\rangle_{k}$, indexed by $\alpha \in \mathbb{C}$, defined as eigenvectors of the annihilation operator $a_{k}: a_{k}|\alpha\rangle_{k}=\alpha|\alpha\rangle_{k}$. The state $|\alpha\rangle_{k}$ is not an eigenvector of the number operator $\widehat{N}=\sum_{k} a_{k}^{*} a_{k}$ and the average of $\widehat{N}$ in $|\alpha\rangle_{k}$ is $|\alpha|^{2}$. The phase $\theta \in(-\pi, \pi]$ of the coherent state, defined by $\alpha=|\alpha| \mathrm{e}^{\mathrm{i} \theta}$, is considered to be a random variable.

2000 Mathematics Subject Classification. 81T27, 81V80, 82C10. 
The action of the displacement operator $D_{k}(\alpha)=\mathrm{e}^{\alpha a_{k}^{*}-\bar{\alpha} a_{k}}$ on the vacuum vector $\Omega$ of the quantum field yields the coherent state: $|\alpha\rangle_{k}=D_{k}(\alpha) \Omega$. In order to produce multimode coherent states, one selects $N$ modes $k_{1}, \ldots, k_{N}$, and defines $D_{k_{1}}\left(\alpha_{1}\right) \cdots D_{k_{N}}\left(\alpha_{N}\right) \Omega$, for any choice of $\alpha_{j} \in \mathbb{C}, j=1, \ldots, N$.

We consider the thermodynamic limit $\Lambda \rightarrow \mathbb{R}^{3}$ of the radiation field. Our main physical motivation for doing this is to thus construct a "large quantum system" which serves as a model for an environment, or reservoir. When we couple a "small system" of "ordinary size" (much smaller than $\Lambda$ ) to the reservoir, then we obtain an open quantum system. On the one hand, the infinite nature of the reservoir causes irreversible dynamical effects in the small system, such as thermalization, decoherence and disentanglement, see Section 1.1. On the other hand, taking the infinite volume limit of the quantum field alone is an interesting mathematical challenge, which uncovers new Hilbert space representations of the canonical commutation relations, see Section 1.2. Similar representations for quantum fields in thermal states (as opposed to coherent states) have been constructed before by Araki and Woods and Araki and Wyss $[\mathbf{1}, \mathbf{2}]$. The resulting thermal representations of the canonical commutation relations have found important applications in mathematical physics.

1.1. Irreversibility and limit of continuous modes. Quantum systems whose dynamics is generated by a Hamiltonian $H$ having purely discrete spectrum

$$
\operatorname{spec}(H)=\left\{E_{j}\right\}_{j \in \mathbb{N}}
$$

show quasi-periodic dynamics. Namely, the expectation of an observable $A$ in a state $\rho(t)=\mathrm{e}^{-\mathrm{i} t H} \rho(0) \mathrm{e}^{\mathrm{i} t H}$ is given by

$$
\langle A\rangle_{t}=\operatorname{Tr} \rho(t) A=\sum_{j, k \in \mathbb{N}} \mathrm{e}^{-\mathrm{i} t\left(E_{k}-E_{l}\right)} c_{k, l},
$$

which is a quasi-periodic function of time $t$. This setup cannot describe irreversible processes, such as the approach to a final state for large times $t \rightarrow \infty$. To capture irreversible effects, one has to consider a limiting situation. An illustrative example is a quantum particle moving freely within a one-dimensional interval $[-L, L]$. The Hamiltonian is $H_{L}=-\frac{d^{2}}{d x^{2}}$ with, say, periodic boundary conditions. The spectrum is purely discrete, $\operatorname{spec}\left(H_{L}\right)=\left\{\frac{\pi^{2} n^{2}}{L^{2}}\right\}_{n \in \mathbb{N}}$ and has gaps of the order $1 / L^{2}$. Averages of observables are oscillating in time and only in the limit $L \rightarrow \infty$ can one observe irreversibility. For instance, suppose that the particle is initially in the state $\rho(0)=$ $|\psi\rangle\langle\psi|$, localized in a bounded region $[-l, l]$ of $\mathbb{R}$ (with $l<L$, so that the support of the wave function $\psi(x)$ is inside $[-l, l])$. Then the probability of finding the particle at time $t$ inside a bounded interval $I \subset \mathbb{R}$ is given by

$$
p_{I}(t)=\int_{I} \mathrm{~d} x\left|\mathrm{e}^{-\mathrm{i} t H_{L}} \psi(x)\right|^{2}
$$

and satisfies $\lim _{t \rightarrow \infty} \lim _{L \rightarrow \infty} p_{I}(t) \rightarrow 0$ (use the Fourier transform to diagonalize $H_{L}$ ). The irreversibility is physically due to the fact that the particle can escape to spatial $\pm \infty$ when $L \rightarrow \infty$, and it is accompanied by the fact that the spectrum of $H_{L}$ becomes continuous in that limit (the gap size $1 / L^{2} \rightarrow 0$ ).

It becomes thus apparent that in order to describe irreversible effects in a quantum system, at least some component of that system should 'become infinitely extended' or show 'continuous energy values'. In the above example, taking the infinite volume limit $L \rightarrow \infty$ is a rather simple procedure. The Hilbert space for 
the confined system is $L^{2}([-L, L], d x)$ (with periodic boundary conditions) and as $L \rightarrow \infty$, the Hilbert space of the infinite system is $L^{2}(\mathbb{R}, \mathrm{d} x)$. The relation between the 'finite $L$ ' and the 'infinite $L$ ' Hilbert spaces can be very complicated though, for other systems of interest.

To see a simple example illustrating this phenomenon, consider a system composed of $K$ independent harmonic quantum oscillators, each one described by its own Hilbert space of pure states $\mathcal{H}_{\text {osc }}$. Such oscillator arrays are ubiquitous in the physics literature on open quantum systems. They model 'reservoirs', components of open systems having many degrees of freedom ( $K$ large). A pure state of the oscillator array is given by a normalized vector in the Hilbert space $\otimes_{j=1}^{K} \mathcal{H}_{\text {osc }}$. Suppose now that the frequencies of the $K$ oscillators are $\omega_{1}, \ldots, \omega_{K}$, and that we want to create a model which shows irreversibility. We must then take $K \rightarrow \infty$ and a frequency spectrum that becomes continuous. What is the Hilbert space of the oscillators in this limit of continuous modes? This question does not have an obvious answer at all. Indeed, in the wanted limit, the cardinality $K$ of the number of modes (frequencies) will increase to $\mathfrak{c}=|\mathbb{R}|$, the cardinality of $\mathbb{R}$. Then we do not even have a candidate for a Hilbert space, since we do not know how to make sense of $\otimes_{j=1}^{K} \mathcal{H}_{\text {osc }}$ in this limit! It turns out that one can construct a Hilbert space for the infinite system following a general procedure, but the Hilbert space will depend on a 'reference state' with respect to which the infinite mode limit is taken.

1.2. Continuous mode limit and reconstruction of a Hilbert space. As discussed in Section 1.1 it is not in general clear how to perform a continuous mode limit. The issue is that such a limit cannot be taken directly on the states (wave functions, density matrices) of a sequence of discrete mode systems. The idea is then to implement the following procedure, which we explain here heuristically and treat in more detail in Sections 2.3 and 2.4.

1. Consider observables $A$ belonging to a (quasi-)local algebra $\mathcal{A}_{0}$ of observables and a sequence of states $\rho_{L}$, with $L$ describing the system 'size' (spatial volume, $1 /$ gap between energy values...).

2. Take the limit $L \rightarrow \infty$ of the average of $A$ in $\rho_{L}$,

$$
\lim _{L \rightarrow \infty}\langle A\rangle_{L} \equiv \lim _{L \rightarrow \infty} \operatorname{Tr}\left(\rho_{L} A\right)=:\langle A\rangle
$$

Assuming the existence of this limit, it defines a state $\langle\cdot\rangle$ on the algebra $\mathcal{A}_{0}$. (Here, a state is defined as a positive, linear, normalized functional on the $C^{*}$-algebra $\mathcal{A}_{0}$ as is usual in algebraic quantum theory.)

3. While we have constructed now the continuous mode limit state $\langle\cdot\rangle$ by the values it takes on the algebra $\mathcal{A}_{0}$ in step 2., we have lost the notion of Hilbert space of the state. However, the general Gelfand-Naimark-Segal reconstruction theorem $[3]$ ensures that to the given pair $\left(\langle\cdot\rangle, \mathcal{A}_{0}\right)$ is assciated a unique triple (the 'GNS triple') $(\mathcal{H}, \pi, \Omega)$ of a Hilbert space $\mathcal{H}$, a $*$-representation map $\pi: \mathcal{A}_{0} \rightarrow \mathcal{B}(\mathcal{H})$ (bounded operators on $\mathcal{H}$ ) and a unit vector $\Omega \in \mathcal{H}$, satisfying

$$
\langle A\rangle=\langle\Omega, \pi(A) \Omega\rangle .
$$

This restores the 'usual' picture of quantum theory, where the state is determined by a unit vector in a Hilbert space. Of course, it is not clear a priori what the concrete realization of the GNS representation Hilbert space is for a given system. For example, Araki and Woods [1] have found the following explicit representation 
for the infinite volume limit $\left(\mathbb{R}^{3}\right)$ of a free bose gas in thermal equilibrium at temperature $T=1 / \beta$ :

$$
\begin{aligned}
\mathcal{H}_{A W} & =\mathcal{F} \otimes \mathcal{F} \\
\pi_{A W}\left(a^{*}(f)\right) & =a^{*}(\sqrt{1+\mu} f) \otimes \mathbb{1}+\mathbb{1} \otimes a(\sqrt{\mu} \bar{f}) \\
\Omega_{A W} & =\Omega \otimes \Omega
\end{aligned}
$$

Here, $\mathcal{F}$ is the Fock space over the one-particle space $L^{2}\left(\mathbb{R}^{3}, d^{3} k\right)$ (Fourier space, see also Section 2.1), $\Omega$ is the vacuum vector in $\mathcal{F}, \mu=\mu(k)=\left(\mathrm{e}^{-\beta \varepsilon(k)}-1\right)^{-1}$ with $k \mapsto \varepsilon(k)$ the dispersion relation, and $a(f), a^{*}(f)$ are the annihilation and creation operators (smoothed out with a form factor $f \in L^{2}\left(\mathbb{R}^{3}\right)$ ). A detailed textbook-style derivation of the Araki-Woods representation is given in [17].

1.3. Why look for a Hilbert space representation? In the physics literature on open quantum systems, it is customary to calculate expressions of interest for discrete mode systems and then take the continuous mode limit of those expressions 'by hand' (e.g. by turning sums over frequencies or energies into corresponding integrals). In this way, the question of the Hilbert space for the continuous mode system is completely avoided. This procedure cannot be carried out rigorously as most often, considerations involve, on top of the continuous mode limit, a limit of weak coupling or long times, making it impossible to control remainder terms. Even more fundamentally, not all quantities of interest have a form for which the abovementioned continuous mode limit 'by inspection' can be found (not all quantities of interest have the convenient form of a 'Riemann sum' over discrete energies which can be turned into an integral in the continuous mode limit, see e.g. [15]). Knowing the continuous mode state itself, by following the GNS procedure we outlined in Section 1.2 (rather than only the expectation of some observables in it), gives in principle full access to the analysis of all quantities. It enables a mathematically rigorous treatment of the dynamics of open quantum systems.

One of the main goals of [11] is to construct the Hilbert space representation of coherent state reservoirs (with continuous modes), similar to the work of Araki and Woods and Araki and Wyss $[\mathbf{1}, \mathbf{2}]$, who did that for thermal Bose and Fermi reservoirs.

1.4. Overview of some of the results in [11]. We provide in this section an informal overview of the results to be stated more precisely below. We consider the quantized radiation field in a finite box $\Lambda \subset \mathbb{R}^{3}$, choose modes $k_{1}, \ldots, k_{N}$ and consider the multimode coherent state $D_{k_{1}}\left(\alpha_{1}\right) \cdots D_{k_{N}}\left(\alpha_{N}\right) \Omega$. Our goal is to analyze this state in the limit $\Lambda \rightarrow \mathbb{R}^{3}$. As $\Lambda$ changes, the eigenmodes of the field change accordingly, and in the limit of infinite volume, the values of the modes becomes a continuum, $k \in \mathbb{R}^{3}$, similarly to the discussion in Section 1.1. It turns out that any state $\omega$ of the radiation field (in finite or infinite volume) is determined uniquely by its expectation functional $E(f)=\omega(W(f))$, where $W(f)$ is the Weyl operator smoothed out with a test function $f$. Thus, it is natural to consider convergence of a sequence of states $\omega_{\Lambda_{n}}$ (with $\Lambda_{n} \rightarrow \mathbb{R}^{3}$ ) to mean convergence of the associated $E_{n}(f)$, for all $f$.

\section{(A) Thermodynamic limit.}

- For $N$ fixed modes $k_{1}, \ldots, k_{N} \in \mathbb{R}^{3}$, we obtain an explicit formula for the limiting state when $\Lambda \rightarrow \mathbb{R}^{3}$ while keeping the particle densities $\rho_{j}=$ $\left|\alpha_{j}\right|^{2} /|\Lambda|$, and the phases $\theta_{j} \in S^{1}$ fixed. 
- A continuous mode limit is characterized by a mode density distribution $\rho(k)$, meaning that $\rho(k) \mathrm{d}^{3} k$ is the spatial density of particles (number of particles per unit volume in direct space) having momenta in an infinitesimal volume $\mathrm{d}^{3} k$ around $k$. We show that the infinite volume and continuous mode limit (taken jointly or not) of the multimode coherent state does not exist.

- Despite the lack of convergence of the expectation functional in the limits of infinite volume and continuous modes, we show that if the phases of the coherent states are random and iid, then the state converges in distribution for the above limits. Mathematically, the convergence is due to the central limit theorem. As explained in Subsection 1.2, we now have the infinite volume, continuous mode state as a functional on a suitable algebra of observables. The next step is to reconstruct a Hilbert space representation for this state.

(B) Hilbert space representation.

We construct explicit Gelfand-Naimark-Segal (GNS) Hilbert spaces of the finitemode infinite volume states, and for the state of continuous modes and random phases. They are regular representations of the canonical commutation relations, defining (represented) field-, creation- and annihilation operators. As mentioned in Section 1.3, the explicit representations provide a rigorous way to analyze the dynamics of a small quantum system coupled to the coherent state reservoir (see point (D) below).

\section{(C) Dynamics of the infinite coherent state.}

The Heisenberg dynamics of the quantum field alone is a Bogoliubov transformation on Weyl operators, $W(f) \mapsto W\left(\mathrm{e}^{\mathrm{i} t \varepsilon} f\right)$, where $t$ denotes time and $\varepsilon=\varepsilon(k)$ is the dispersion relation. Coherent states are not eigenstates of this dynamics and so the reservoir has a nontrivial dynamics on its own. We show that the random iid phase state is driven into a final state for large times. The latter corresponds to an iid random phase state in which the phases are uniformly distributed. In this sense, the uniform phase distribution is the stable one.

\section{(D) Coupling to an open quantum system.}

We consider an $N$-level quantum system in contact with the infinite volume coherent reservoir having uniformly randomly distributed phases, the dynamically stable reservoir state as explained in point $(\mathrm{C})$ above. We consider an energy conserving coupling between the $N$-level system and the reservoir so that the interaction term in the Hamiltonian describing the coupled evolution commutes with the system Hamiltonian. This model is explicitly solvable in the sense that we can calculate the exact density matrix of the $N$-level system at all times. We find that the expectation of the off-diagonal density matrix elements (in the energy basis) have Gaussian time decay due to the coupling with the coherent reservoir. This is a striking difference relative to the thermal reservoir case, where this decay is only exponential. We conclude that a small system placed in a random phase coherent reservoir undergoes much faster decoherence than in a thermal environment. ${ }^{1}$ The

\footnotetext{
${ }^{1}$ The name "coherent states" refers to the quantum field and is motivated by the fact that correlation functions of the field factorize in those states, which is the same as for classical coherent fields [13]. On the other hand "decoherence" of a quantum system is an entirely different notion, which refers to a system losing quantum correlations and becoming close to a classical one [10].
} 
very rapid loss of "quantumness" encoded by coherence of the small system is yet another manifestation of the classical character of coherent states.

Besides the energy conserving coupling considered here, which serves as a benchmark to measure the decay of coherence in the small system, it is desirable to analyze the dynamics of an $N$-level system coupled in a generic, not necessarily energy conserving fashion to the infinite random coherent reservoir. This turns out to be a difficult task. However, if one is interested in specific properties of the coupled system in an average sense with respect to the randomness of the reservoir only, one might hope for certain simplifications to occur when taking expectation values over the randomness. This hope is substantiated by the fact that the randomness in the reservoir state is completely captured by a Gaussian complex valued random variable.

\section{Setup and main results}

We now turn to the description of the general setup and concrete statements. Consider non-interacting quantum particles confined to a box of sidelength $L$ in $d$ dimensions,

$$
\Lambda=[-L / 2, L / 2]^{d} \subset \mathbb{R}^{d}
$$

The wave function of a single particle is an element of $L^{2}(\Lambda, \mathrm{d} x)$ with periodic conditions at the boundaries. The space of pure states of the system of particles is the symmetric Fock space $[\mathbf{3}]$

$$
\mathcal{F} \equiv \mathcal{F}\left(L^{2}(\Lambda, \mathrm{d} x)\right)=\bigoplus_{n \geq 0} L_{\text {symm }}^{2}\left(\Lambda^{n}, \mathrm{~d}^{n} x\right) .
$$

Elements of $\mathcal{F}$ have the form $\psi=\oplus_{n \geq 0} \psi^{(n)}$, where $\psi^{(n)}\left(x_{1}, \ldots, x_{n}\right)$ is a symmetric function of $n$ variables $x_{j} \in \mathbb{R}^{d}$ which is periodic in $\Lambda^{n}$. The summand $n=0$ of the Fock space is called the vacuum sector, spanned by the vacuum vector $\Omega$ characterized by $\Omega^{(0)}=1, \Omega^{(n)}=0$ for $n \geq 1$.

The generator of the Schrödinger equation

$$
\psi_{t}=\mathrm{e}^{-\mathrm{i} H t} \psi_{0},
$$

is the self-adjoint Hamiltonian given by the second quantization of a one-body Hamilton operator. For photons (massless relativistic particles), the Hamiltonian writes

$$
(H \psi)^{(n)}\left(x_{1}, \ldots, x_{n}\right)=\sum_{j=1}^{n} \sqrt{-\Delta_{x_{j}}} \psi^{(n)}\left(x_{1}, \ldots, x_{n}\right)
$$

understood as a self-adjoint operator with periodic boundary conditions.

The creation operator $a^{*}(f)$ is defined for $f \in L^{2}(\Lambda, \mathrm{d} x)$ by

$$
\left(a^{*}(f) \psi\right)^{(n)}\left(x_{1}, \ldots, x_{n}\right)=\sqrt{n} \mathcal{S} f\left(x_{1}\right) \psi^{(n-1)}\left(x_{2}, \ldots, x_{n}\right),
$$

where $\mathcal{S}$ is the operator of symmetrization over the variables $x_{1}, \ldots, x_{n}$. The $a n$ nihilation operator $a(f)$, given by

$$
(a(f) \psi)^{(n)}\left(x_{1}, \ldots, x_{n}\right)=\sqrt{n+1} \int_{\Lambda} \bar{f}(x) \psi^{(n+1)}\left(x, x_{1}, \ldots, x_{n}\right) \mathrm{d} x,
$$

is the adjoint of the creation operator. We also introduce the the self-adjoint field operators

$$
\Phi(f)=\frac{1}{\sqrt{2}}\left(a^{*}(f)+a(f)\right)
$$


and the unitary Weyl operators

$$
W(f)=\mathrm{e}^{\mathrm{i} \Phi(f)}
$$

The following equivalent canonical commutation relations hold:

$$
\begin{aligned}
{\left[a(f), a^{*}(g)\right] } & =\langle f, g\rangle \\
{[\Phi(f), \Phi(g)] } & =\mathrm{i} \operatorname{Im}\langle f, g\rangle \\
W(f) W(g) & =\mathrm{e}^{-\frac{\mathrm{i}}{2} \operatorname{Im}\langle f, g\rangle} W(f+g) .
\end{aligned}
$$

A normalized vector $\psi$ in $\mathcal{F}$ determines an expectation functional, defined by $E(f):=\langle\psi, W(f) \psi\rangle, f \in L^{2}(\Lambda, \mathrm{d} x)$. Conversely, any functional $E: L^{2}(\Lambda, \mathrm{d} x) \rightarrow \mathbb{C}$ satisfying the three conditions

(E1) $E(0)=1$

(E2) $\overline{E(f)}=E(-f)$

(E3) $\sum_{k, k^{\prime}=1}^{K} z_{k} \overline{z_{k^{\prime}}} \mathrm{e}^{\frac{\mathrm{i}}{2} \operatorname{Im}\left\langle f_{k}, f_{k^{\prime}}\right\rangle} E\left(f_{k}-f_{k^{\prime}}\right) \geq 0$, for all $K \geq 1, z_{k} \in \mathbb{C}, f_{k} \in$ $L^{2}(\Lambda, \mathrm{d} x)$

determines a state $\rho$ on the $C^{*}$-algebra generated by the Weyl operators by the relation $\rho(W(f))=E(f)$, see e.g. $[\mathbf{1 7}]$.

We pass to the momentum space representation, the Fourier transformation of the Fock space $\mathcal{F}$, in order to define coherent states of the particles in the volume $\Lambda$.

2.1. Momentum space representation. The single-particle Hilbert space $L^{2}(\Lambda, \mathrm{d} x)$ is unitarily equivalent to $l^{2}\left(\frac{2 \pi}{L} \mathbb{Z}^{d}\right)$ via the Fourier transform $\mathfrak{F}: L^{2}(\Lambda, \mathrm{d} x) \rightarrow$ $l^{2}\left(\frac{2 \pi}{L} \mathbb{Z}^{d}\right)$

$$
(\mathfrak{F} f)(k)=\widehat{f}_{k}=L^{-d / 2} \int_{\Lambda} \mathrm{e}^{-\mathrm{i} k x} f(x) \mathrm{d} x,
$$

with inverse

$$
\left(\mathfrak{F}^{-1} \widehat{f}\right)(x)=L^{-d / 2} \sum_{k \in \frac{2 \pi}{L} \mathbb{Z}^{d}} \mathrm{e}^{\mathrm{i} k x} \widehat{f}_{k} .
$$

The expression, $k x$ denotes the dot product $k \cdot x$ and the factors $L^{-d / 2}$ guarantee that $\mathfrak{F}$ is unitary. In turn, the Fock space $\mathcal{F}$ is unitarily equivalent to its momentum version

$$
\widehat{\mathcal{F}} \equiv \mathcal{F}\left(l^{2}\left(\frac{2 \pi}{L} \mathbb{Z}^{d}\right)\right)=\bigoplus_{n \geq 0}\left(l^{2}\left(\frac{2 \pi}{L} \mathbb{Z}^{d}\right)\right)^{\otimes_{\mathrm{symm}}^{n}}
$$

with $\Omega$ and $\widehat{\Omega}$, the vacua of the Fock spaces $\mathcal{F}$ and $\widehat{\mathcal{F}}$, respectively. The unitary map between the Fock spaces is characterized by

$$
\mathfrak{F} a^{*}\left(f_{1}\right) \cdots a^{*}\left(f_{\ell}\right) \Omega=a^{*}\left(\widehat{f}_{1}\right) \cdots a^{*}\left(\widehat{f}_{\ell}\right) \widehat{\Omega} .
$$

Accordingly, the creation operators transform as $\mathfrak{F} a^{*}(f) \mathfrak{F}^{-1}=a^{*}(\widehat{f})$ and we write

$$
a^{*}(\widehat{f})=\sum_{k \in \frac{2 \pi}{L} \mathbb{Z}^{d}} \widehat{f}_{k} a_{k}^{*}
$$

The field- and Weyl operators are transported to the momentum space as

$$
\mathfrak{F} \Phi(f) \mathfrak{F}^{-1}=\Phi(\widehat{f}) \quad \text { and } \quad \mathfrak{F} W(f) \mathfrak{F}^{-1}=W(\widehat{f}),
$$

where $\Phi(\widehat{f})=\frac{1}{\sqrt{2}} \sum_{k \in \frac{2 \pi}{L} \mathbb{Z}^{d}}\left(\widehat{f}_{k} a_{k}^{*}+{\widehat{f_{k}}}_{k}\right)$ and $W(\widehat{f})=\mathrm{e}^{\mathrm{i} \Phi(\widehat{f})}$. 
2.2. $N$-mode coherent states in finite volume. In line with Section 1.4, the coherent state associated to the collection of $N$ modes $k_{1}^{\prime}, \ldots, k_{N}^{\prime} \in \frac{2 \pi}{L} \mathbb{Z}^{d}$ and $N$ complex numbers $\alpha_{1}, \ldots, \alpha_{N}$ is the normalized vector

$$
\widehat{\Psi}=\mathrm{e}^{\sum_{j=1}^{N} \alpha_{j} a_{k_{j}^{\prime}}^{*}-\bar{\alpha}_{j} a_{k_{j}^{\prime}} \widehat{\Omega} \in \widehat{\mathcal{F}} .}
$$

The expectation functional corresponding to the coherent state (2.3) is thus

$$
E_{N}^{\Lambda}(f)=\langle\widehat{\Psi}, W(\widehat{f}) \widehat{\Psi}\rangle
$$

for all $\widehat{f} \in l^{2}\left(\frac{2 \pi}{L} \mathbb{Z}^{d}\right)$. The number operator $a_{k_{j}^{\prime}}^{*} a_{k_{j}^{\prime}}$ of the mode $k_{j}^{\prime} \in \frac{2 \pi}{L} \mathbb{Z}^{d}$ has average in the coherent state given by

$$
\left\langle\widehat{\Psi}, a_{k_{j}^{\prime}}^{*} a_{k_{j}^{\prime}} \widehat{\Psi}\right\rangle=\left|\alpha_{j}\right|^{2}
$$

which is interpreted as the intensity of the mode considered.

\subsection{Infinite volume and continuous mode limits, random phases.}

2.3.1. Infinite volume. The momenta in the finite-volume coherent state are of the form $k_{j}^{\prime}=2 \pi n_{j} / L$, for $n_{j} \in \mathbb{Z}^{d}$, so that as $L$ increases, their spacing becomes increasingly small. Consider now $k_{1}, \ldots, k_{N} \in \mathbb{R}^{d}, N$ arbitrary target momenta and take $n_{j}=n_{j}(L) \in \mathbb{Z}^{d}$ so that $k_{j}^{\prime}(L)=2 \pi n_{j}(L) / L$ satisfies $\lim _{L \rightarrow \infty} k_{j}^{\prime}(L)=k_{j}$, $j=1, \ldots, N$. We want to take the thermodynamic limit of $(2.4)$,

$$
\lim _{L \rightarrow \infty} E_{N}^{\Lambda}(f) \equiv E_{N}(f) .
$$

This means we take $\lim _{L \rightarrow \infty} k_{j}^{\prime}=k_{j}^{\prime}(L)$, while keeping fixed the particle densities $\rho_{j} \geq 0$ which count the (average) number of particles in mode $k_{j}^{\prime}$ per unit volume, for $j=1, \ldots, N$. This imposes $\left|\alpha_{j}\right|^{2}=L^{d} \rho_{j}$, or

$$
\alpha_{j}(L)=L^{d / 2} \sqrt{\rho_{j}} \mathrm{e}^{\mathrm{i} \theta_{j}},
$$

where $\theta_{j}$ is the phase of the complex number $\alpha_{j}$, which is arbitrary so far.

Proposition 2.1 (Thermodynamic limit for $N$ modes, $[\mathbf{1 1}]$ ). Let $k_{1}, \ldots, k_{N} \in$ $\mathbb{R}$ and $\rho_{1}, \ldots, \rho_{N} \geq 0$ be arbitrary momenta and arbitrary particle densities and suppose that $f \in L^{1}\left(\mathbb{R}^{d}, \mathrm{~d} x\right) \cap L^{2}\left(\mathbb{R}^{d}, \mathrm{~d} x\right)$. Then the limit (2.5) exists and

$$
E_{N}(f)=E_{\text {Fock }}(f) \mathrm{e}^{\mathrm{i} \operatorname{Re} \sum_{j=1}^{N} \mathrm{e}^{-\mathrm{i} \theta_{j}} \sqrt{2 \rho_{j}} \widehat{f}\left(k_{j}\right)},
$$

where $E_{\text {Fock }}(f)=\mathrm{e}^{-\frac{1}{4}\|f\|^{2}}$ and $\widehat{f}(k)=\int_{\mathbb{R}^{d}} \mathrm{e}^{-\mathrm{i} k x} f(x) \mathrm{d} x$.

Here, $E_{\text {Fock }}(f)$ is the usual Fock expectation functional, determined by the vacuum state,

$$
E_{\text {Fock }}(f)=\langle\Omega, W(f) \Omega\rangle=\mathrm{e}^{-\frac{1}{4}\|f\|_{2}^{2}}=\mathrm{e}^{-\frac{1}{4}(2 \pi)^{-d}\|\widehat{f}\|_{2}^{2}},
$$

where $\|\cdot\|_{2}$ is the $L^{2}$-norm (of functions of $k \in \mathbb{R}^{d}$ or $x \in \mathbb{R}^{d}$ ),

$$
\|f\|_{2}^{2}=\int_{\mathbb{R}^{d}}|f(x)|^{2} \mathrm{~d} x \quad \text { and } \quad\|\widehat{f}\|_{2}^{2}=\int_{\mathbb{R}^{d}}|\widehat{f}(k)|^{2} \mathrm{~d} k .
$$


2.3.2. Continuous modes. One may perform the infinite-volume limit and the continuous mode limit simultaneously, or one can take the continuous mode limit of (2.7). The result is the same [11] and we do the latter. Let $\rho(k)$ be a prescribed mode density distribution. Therefore, given a cube $I \subset \mathbb{R}^{d}$, the integral $\int_{I} \rho(k) \mathrm{d} k$ is the spatial density of particles in the infinite volume state, having momenta in $I$. Assume $\rho$ is be supported in a finite cube $[-R, R]^{d}$. Discretize the cube by setting $k_{j}=\left(-R+j_{1} \frac{2 R}{N}, \ldots,-R+j_{d} \frac{2 R}{N}\right) \in \mathbb{R}^{d}$, where $j_{1}, \ldots, j_{d} \in\{1,2, \ldots, N\}$, and $N$ is large. One gets the following form for the sum in the phase of the infinite volume expectation functional (2.7),

$$
\sum_{j \in\{1, \ldots, N\}^{d}} \mathrm{e}^{-\mathrm{i} \theta_{j}} \sqrt{2 \rho_{j}} \widehat{f}\left(k_{j}\right)=(2 R / N)^{d / 2} \sum_{j \in\{1, \ldots, N\}^{d}} \mathrm{e}^{-\mathrm{i} \theta\left(k_{j}\right)} \sqrt{2 \rho\left(k_{j}\right)} \widehat{f}\left(k_{j}\right) .
$$

Here, $\theta(k)$ is an arbitrary function which determines the phase of the mode $k$. For $N$ large, a Riemann sum argument yields that (2.8) equals approximately

$$
(N / 2 R)^{d / 2} \int_{[-R, R]^{d}} \mathrm{e}^{-\mathrm{i} \theta(k)} \sqrt{2 \rho(k)} \widehat{f}(k) \mathrm{d} k \sim N^{d / 2},
$$

which diverges as $N \rightarrow \infty$, and so the infinite volume discrete mode expectation functional $E_{N}(f),(2.7)$, does not have a continuous mode limit in this simple sense.

2.3.3. Random phases. Considering the scaling involved, one sees that the central limit theorem might help in case the phases are independent random variables: Let $\theta_{j}=\theta_{j}(\omega), j \in\{1, \ldots, N\}^{d}$ be iid random variables, so that $\widehat{\Psi}=\widehat{\Psi}_{\omega}$ given in (2.3) is a random pure state defining the random expectation functional (in infinite volume, by Proposition 2.1)

$$
E_{N, \omega}(f)=E_{\mathrm{Fock}}(f) e^{\mathrm{i} N^{-d / 2} \sum_{j \in\{1, \ldots, N\}^{d}} \xi_{j}(\omega)},
$$

where

$$
\xi_{j}(\omega)=(2 R)^{d / 2} \sqrt{2 \rho\left(k_{j}\right)} \operatorname{Re}^{-\mathrm{i} \theta_{j}(\omega)} \widehat{f}\left(k_{j}\right) .
$$

We know from (2.9) that $E_{N, \omega}(f)$ does not converge almost everywhere w.r.t. $\omega$ as $N \rightarrow \infty$. However, we have the following result.

Proposition $2.2([\mathbf{1 1}])$. Let $\mu$ be the distribution function of the phase and suppose that its Fourier transform satisfies $\widehat{\mu}(1)=0$. Then, in the sense of convergence in distribution,

$$
N^{-d / 2} \sum_{j \in\{1, \ldots, N\}^{d}} \xi_{j}(\omega) \stackrel{\mathcal{D}}{\longrightarrow} \mathcal{N}_{\omega}\left(0, \sigma_{\mu}(f)^{2}\right), \quad \text { as } N \rightarrow \infty .
$$

The right hand side is a normal random variable with mean zero and variance

$$
\sigma_{\mu}(f)^{2}=\int_{\mathbb{R}^{d}} \rho(k)\left(|\widehat{f}(k)|^{2}+\operatorname{Re}\left\{\widehat{\mu}(2) \widehat{f}(k)^{2}\right\}\right) \mathrm{d} k .
$$

The function $x \mapsto \mathrm{e}^{\mathrm{i} x}$ is bounded and continuous and so (2.10) implies that

$$
E_{N, \omega}(f) \stackrel{\mathcal{D}}{\longrightarrow} E_{\omega}(f) \equiv E_{\text {Fock }}(f) \mathrm{e}^{\mathrm{i} \mathcal{N}_{\omega}\left(0, \sigma_{\mu}(f)^{2}\right)}, \quad \text { as } N \rightarrow \infty .
$$

Since the convergence in Proposition 2.2 is only in distribution, it is not guaranteed that $E_{\omega}$ defined in (2.14) satisfies properties (E1)-(E3) which are necessary for $E_{\omega}$ to define a state. Among all random functionals having the distribution given by the right hand side of (2.14), we must make sure that there is one which 
satisfies (E1)-(E3). We construct such an explicit representation by means of Itô stochastic integrals.

For $f \in L^{2}\left(\mathbb{R}^{d}, \mathrm{~d} x\right)$, we define $\chi_{\omega}$ as an Itô integral

$$
\chi_{\omega}(f)=\int_{\mathbb{R}^{d}} \mathrm{~d} B_{1}^{\omega}(k) S_{1}(k) \widehat{f}(k)+\mathrm{i} \int_{\mathbb{R}^{d}} \mathrm{~d} B_{2}^{\omega}(k) S_{2}(k) \widehat{f}(k),
$$

where

$$
S_{1}(k)=\sqrt{\frac{\rho(k)}{1+\operatorname{Re} \widehat{\mu}(2)}}(1+\widehat{\mu}(2)), \quad S_{2}(k)=\sqrt{\frac{\rho(k)}{1+\operatorname{Re} \widehat{\mu}(2)}} \sqrt{1-|\widehat{\mu}(2)|^{2}} .
$$

Here, $B_{1}^{\omega}$ and $B_{2}^{\omega}$ are two independent Brownian motions of dimension $d$ and $\widehat{\mu}$ is the Fourier transform of the measure $\mu$.

THEOREM $2.3([\mathbf{1 1}])$. Suppose that $\widehat{\mu}(1)=0$. Then

(1) For all $f \in L^{2}\left(\mathbb{R}^{d}, \mathrm{~d} k\right)$ we have $\operatorname{Re} \chi_{\omega}(f) \sim \mathcal{N}_{\omega}\left(0, \sigma_{\mu}(f)^{2}\right)$, where $\sigma_{\mu}(f)^{2}$ is given in (2.13).

(2) Let $E_{N, \omega}(f)$ be the functional (2.10). Then we have, for all $f \in L^{2}\left(\mathbb{R}^{d}, \mathrm{~d} x\right)$,

$$
E_{N, \omega}(f) \stackrel{\mathcal{D}}{\longrightarrow} E_{\omega}(f):=E_{\text {Fock }}(f) \mathrm{e}^{\mathrm{i} \operatorname{Re} \chi_{\omega}(f)}, \quad \text { as } N \rightarrow \infty .
$$

(3) $E_{\omega}(\cdot)$ satisfies (E1)-(E3) in the following sense:

$$
\begin{aligned}
& \text { - } E_{\omega}(0)=1 \text { a.e. }(\omega) \\
& \text { - for all } f \in L^{2}\left(\mathbb{R}^{d}, d x\right), \overline{E_{\omega}(f)}=E_{\omega}(-f) \text { a.e. }(\omega) \\
& \text { - for all } K \geq 1, z_{k} \in \mathbb{C}, f_{k} \in L^{2}\left(\mathbb{R}^{d}, d x\right), k=1, \ldots, K, \text { we have } \\
& \sum_{k, k^{\prime}=1}^{K} z_{k} \overline{z_{k^{\prime}}} \mathrm{e}^{\frac{i}{2} \operatorname{Im}\left\langle f_{k}, f_{k^{\prime}}\right\rangle} E_{\omega}\left(f_{k}-f_{k^{\prime}}\right) \geq 0 \text { a.e. }(\omega)
\end{aligned}
$$

Remark. For each $f$ we have $E_{\omega}(f) \in L^{2}(\mathcal{O}, \mathrm{d} \mathbb{P})$, where we call the sample space $\mathcal{O}$. So there is an $\mathcal{O}_{f} \subseteq \mathcal{O}$ with $\mathbb{P}\left(\mathcal{O}_{f}\right)=1$ s.t. $E_{\omega}(f) \in \mathbb{C}$ for all $\omega \in \mathcal{O}_{f}$. (That is, we can choose a representative of the $L^{2}$ function which is well defined and finite on a set of full measure.) Given $f_{1}, \ldots, f_{K}$ we thus find $\mathcal{O}_{f_{1}}, \ldots, \mathcal{O}_{f_{K}}$, all of full measure, so that $\sum_{j=1}^{k} E_{\omega}\left(f_{j}\right)$ is well defined and finite for all $\omega \in \cap_{j=1}^{K} \mathcal{O}_{f_{j}}$, again a set of full measure. The latter sum then defines again an element in $L^{2}(\mathcal{O}, \mathrm{dP})$. In this sense, we can form finite (or countably infinite) linear combinations of $E_{\omega}\left(f_{j}\right)$. The set of $\omega$ of full measure on which (E3) above holds generally depends on the functions $f_{j}$.

2.4. Hilbert space representation. Given a state $\rho$ on a $C^{*}$-algebra $\mathfrak{A}$, there exists a unique (up to unitary equivalence) GNS triple $(\mathcal{H}, \pi, \Psi)[\mathbf{3}]$ consisting of a Hilbert space $\mathcal{H}$, a representation map $\pi: \mathfrak{A} \rightarrow \mathcal{B}(\mathcal{H})$ and a normalized and cyclic vector $\Psi \in \mathcal{H}$, such that for all $A \in \mathfrak{A}$,

$$
\rho(A)=\langle\Psi, \pi(A) \Psi\rangle .
$$

Consider now the family $E_{\omega}$ given in Theorem 2.3, (2). Being an element of $L^{2}(\mathcal{O}, \mathrm{d} \mathbb{P}), E_{\omega}(f)$ is only well defined (represented by a function with finite complex values) for $\omega \in \mathcal{O}_{f} \subseteq \mathcal{O}$ for some $\mathcal{O}_{f}$ with $\mathbb{P}\left(\mathcal{O}_{f}\right)=1$. The range over which $\omega$ varies thus depends on $f$. Therefore, it is not clear that there is any $\omega \in \mathcal{O}$ for which one can define simultaneously $E_{\omega}(f)$ for all $f \in L^{2}\left(\mathbb{R}^{d}, \mathrm{~d} x\right)$. However, we can restrict the range of $f$ to a countable subset of "test functions" $\mathcal{D} \subset L^{2}\left(\mathbb{R}^{d}, \mathrm{~d} x\right)$. For each $f \in \mathcal{D}$, there is an $\mathcal{O}_{f} \subseteq \mathcal{O}, \mathbb{P}\left(\mathcal{O}_{f}\right)=1$, on which $E_{\omega}(f)$ is well defined, 
that is, for which one can choose a representative of the $L^{2}(\mathcal{O}, \mathrm{d} \mathbb{P})$ function which is finite on $\mathcal{O}_{f}$. Being a countable intersection of sets of measure one, the set

$$
\mathcal{O}(\mathcal{D})=\cap_{f \in \mathcal{D}} \mathcal{O}_{f}
$$

has also measure one. Furthermore, for every $\omega \in \mathcal{O}(\mathcal{D})$ fixed, $E_{\omega}(f)$ is well defined for all $f \in \mathcal{D}$. Without loss of generality, we may assume that $\mathcal{D}$ is a vector space over the countable field $\mathbb{Q}+i \mathbb{Q}$. (If an original $\mathcal{D}$ is not, then we can consider the set of all finite linear combinations of elements of $\mathcal{D}$, with scalars from $\mathbb{Q}+\mathrm{i} \mathbb{Q}$. This is again a countable set and we can take that set for $\mathcal{D}$.)

We show in $[\mathbf{1 1}]$ that there exists an $\mathcal{O}(\mathcal{D}) \subseteq \mathcal{O}$ of full measure, such that $\forall \omega \in \mathcal{O}(\mathcal{D}), E_{\omega}$ is an expectation functional on the Weyl algebra with test functions $f \in \mathcal{D}$, i.e., the Weyl algebra (over the field $\mathbb{C}$ of scalars) generated by all $W(f)$, $f \in \mathcal{D}$.

THEOREM 2.4 (GNS representation $[\mathbf{1 1}])$. Let $\mathcal{D} \subset L^{2}\left(\mathbb{R}^{d}, \mathrm{~d} x\right)$ be a test function subspace with associated $\mathcal{O}(\mathcal{D})$ satisfying $\mathbb{P}(\mathcal{O}(\mathcal{D}))=1$. For every $\omega \in \mathcal{O}(\mathcal{D})$, the GNS representation of $E_{\omega}(\cdot)$ (c.f. Theorem 2.3(2)) as a functional of the Weyl algebra with test functions in $\mathcal{D}$, is given by

$$
\begin{aligned}
\mathcal{H} & =\mathcal{H}_{\mathcal{D}} \subseteq \mathcal{F}\left(L^{2}\left(\mathbb{R}^{d}, \mathrm{~d} x\right)\right) \\
\pi_{\omega}(W(f)) & =W_{\text {Fock }}(f) \mathrm{e}^{\mathrm{iRe} \chi_{\omega}(f)} \\
\Psi & =\Omega_{\text {Fock }} .
\end{aligned}
$$

Recall that a representation $\pi$ is called regular if $\alpha \mapsto \pi(W(\alpha f))$ is differentiable at $\alpha=0$, in the strong sense on a dense domain in $\mathcal{H}$. For regular representations, one defines the represented Weyl operators

$$
W_{\pi}(f)=\pi(W(f))
$$

and the represented field operators by

$$
\Phi_{\pi}(f)=-\left.i \partial_{\alpha}\right|_{\alpha=0} \pi(W(\alpha f)) .
$$

Similarly, the creation and annihilation operators are defined by

$$
\begin{aligned}
& a_{\pi}^{*}(f)=2^{-1 / 2}\left[\Phi_{\pi}(f)-\mathrm{i} \Phi_{\pi}(\mathrm{i} f)\right], \\
& a_{\pi}(f)=2^{-1 / 2}\left[\Phi_{\pi}(f)+\mathrm{i} \Phi_{\pi}(\mathrm{i} f)\right]=\left(a_{\pi}^{*}(f)\right)^{*} .
\end{aligned}
$$

The representation of Theorem 2.4 is actually regular, so that the field and creation operators associated to $E_{\omega}$, for all $\omega \in \mathcal{O}(\mathcal{D})$ and all $f \in \mathcal{D}$, are given by

$$
\begin{aligned}
\Phi_{\omega}(f) & =\Phi_{\text {Fock }}(f)+\operatorname{Re} \chi_{\omega}(f) \\
a_{\omega}^{*}(f) & =a_{\text {Fock }}^{*}(f)+\frac{1}{\sqrt{2}} \chi_{\omega}(f) .
\end{aligned}
$$

2.5. Reservoir dynamics. We can now address the dynamical aspects of our construction. The dynamics on the Weyl algebra is given by a Bogoliubov transformation on the functions $f \in L^{2}\left(\mathbb{R}^{d}, \mathrm{~d} k\right)$, given by $f \mapsto \mathrm{e}^{\mathrm{i} t \varepsilon} f$, where $\varepsilon=\varepsilon(k)$ is a real function of $k \in \mathbb{R}^{d}$. For photons, $\varepsilon(k)=|k|$. Thus, the dynamics of the random phase expectation functional $E_{\omega},(2.14)$ satisfies

$$
E_{\omega}\left(\mathrm{e}^{\mathrm{i} t \varepsilon} f\right)=E_{\text {Fock }}(f) \mathrm{e}^{\mathrm{i} \mathcal{N}_{\omega}\left(0, \sigma_{\mu}\left(\mathrm{e}^{\mathrm{i} t \varepsilon} f\right)^{2}\right)} .
$$

Now, the Riemann-Lebesgue Lemma and (2.13) show that

$$
\lim _{t \rightarrow \infty} \sigma_{\mu}\left(\mathrm{e}^{\mathrm{i} t \varepsilon} f\right)^{2}=\|\sqrt{\rho} \widehat{f}\|_{2}^{2}
$$


and therefore,

$$
\mathcal{N}_{\omega}\left(0, \sigma_{\mu}\left(\mathrm{e}^{\mathrm{i} t \varepsilon} f\right)^{2}\right) \stackrel{\mathcal{D}}{\longrightarrow} \mathcal{N}_{\omega}\left(0,\|\sqrt{\mu} \widehat{f}\|_{2}^{2}\right), \quad t \rightarrow \infty .
$$

Consequently, we reach the following conclusion:

Proposition 2.5 (Phase uniformization under reservoir dynamics, [11]). Let $\mu$ be a phase distribution satisfying $\widehat{\mu}(1)=0$. Given any $f \in L^{2}\left(\mathbb{R}^{d}, \mathrm{~d} x\right)$, we have, as $t \rightarrow \infty$,

$$
E_{\omega}\left(\mathrm{e}^{\mathrm{i} t \varepsilon} f\right) \stackrel{\mathcal{D}}{\longrightarrow} E_{\omega, \text { unif }}(f) .
$$

The convergence in $(2.21)$ is in distribution of random variables. Here, $E_{\omega, \text { unif }}(\cdot)$ is the expectation functional in which the phase distribution is uniform, $\mathrm{d} \mu(\theta)=\frac{\mathrm{d} \theta}{2 \pi}$.

2.6. Coupling to an open quantum system. We are now in a position allowing us to consider an $N$-dimensional quantum system in contact with the reservoir of coherent states. We consider that the phases are uniformly randomly distributed. The Hilbert space of pure states of the system is $\mathbb{C}^{N}$, that of the reservoir is the GNS space given in Theorem 2.4. The system dynamics is generated by a self-adjoint Hamiltonian with energy levels $e_{1}, \ldots, e_{N}$,

$$
H_{\mathrm{S}}=\operatorname{diag}\left(e_{1}, \ldots, e_{N}\right),
$$

and the state of the reservoir is invariant under its own dynamics by Proposition 2.5. The dynamics is implemented as

$$
\pi_{\omega}\left(W\left(\mathrm{e}^{\mathrm{i} t \varepsilon} f\right)\right)=W_{\text {Fock }}\left(\mathrm{e}^{\mathrm{i} t \varepsilon} f\right) \mathrm{e}^{\mathrm{i} R e \chi_{\omega}(f)}=\mathrm{e}^{\mathrm{i} t H_{\mathrm{R}}} \pi_{\omega}(W(f)) \mathrm{e}^{-\mathrm{i} t H_{\mathrm{R}}},
$$

where the reservoir Hamiltonian is

$$
H_{\mathrm{R}}=\mathrm{d} \Gamma(\varepsilon) .
$$

Therefore, the uncoupled dynamics is given by the Hamiltonian

$$
H_{0}=H_{\mathrm{S}} \otimes \mathbb{1}_{\mathrm{R}}+\mathbb{1}_{\mathrm{S}} \otimes H_{\mathrm{R}} .
$$

To define a coupled dynamics between the system and the reservoir, one makes use of Dyson's expansion. The free dynamics is given by the group of *automorphisms $\alpha_{0}^{t}$ on the algebra of observables $\mathfrak{A}=\mathcal{B}\left(\mathbb{C}^{N}\right) \otimes \mathcal{W}$ (where $\mathcal{W}$ is the Weyl algebra), defined by

$$
\alpha_{0}^{t}\left(A_{\mathrm{S}} \otimes W(f)\right)=\mathrm{e}^{\mathrm{i} t H_{\mathrm{S}}} A_{\mathrm{S}} \mathrm{e}^{-\mathrm{i} t H_{\mathrm{S}}} \otimes W\left(\mathrm{e}^{\mathrm{i} t \varepsilon} f\right) .
$$

Then one defines a coupled dynamics by specifying an interaction operator $V \in \mathfrak{A}$ and using the Dyson series

$$
\alpha^{t}(A)=\alpha_{0}^{t}(A)+\sum_{n \geq 1} \int_{0}^{t} \mathrm{~d} t_{1} \cdots \int_{0}^{t_{n}-1} \mathrm{~d} t_{n}\left[\alpha_{0}^{t_{n}}(V),\left[\cdots\left[\alpha_{0}^{t_{1}}(V), \alpha_{0}^{t}(A)\right] \cdots\right]\right] .
$$

It turns out the series converges in the topology of $\mathfrak{A}$ and thus defines the interacting dynamics $\alpha^{t}$, again a group of $*$ automorphisms on $\mathfrak{A}$. Applying the representation map $\pi_{\omega}$ (more precisely, $\mathbb{1}_{\mathrm{S}} \otimes \pi_{\omega}$ ) to $(2.22)$, we obtain

$$
\begin{aligned}
& \pi_{\omega}\left(\alpha^{t}(A)\right)=\tau_{0}^{t}\left(\pi_{\omega}(A)\right) \\
& \quad+\sum_{n \geq 1} \int_{0}^{t} \mathrm{~d} t_{1} \cdots \int_{0}^{t_{n}-1} \mathrm{~d} t_{n}\left[\tau_{0}^{t_{n}}\left(\pi_{\omega}(V)\right),\left[\cdots\left[\tau_{0}^{t_{1}}\left(\pi_{\omega}(V)\right), \tau_{0}^{t}\left(\pi_{\omega}(A)\right)\right] \cdots\right]\right],
\end{aligned}
$$

where

$$
\tau_{0}^{t}(\cdot)=\mathrm{e}^{\mathrm{i} t H_{0}}(\cdot) \mathrm{e}^{-\mathrm{i} t H_{0}}
$$


The right side of $(2.23)$ defines a group of $*$ automorphisms on the represented algebra of observables which is generated by the self-adjoint operator

$$
H=H_{0}+\pi_{\omega}(V)
$$

acting on $\mathbb{C}^{N} \otimes \mathcal{F}\left(L^{2}\left(\mathbb{R}^{d}, \mathrm{~d} x\right)\right)$. Physical considerations would lead us to take $V=$ $G \otimes \Phi(g)$, where $G$ is selfadjoint and $\Phi(g)$ is a field operator. However, this $V$ does not belong to $\mathfrak{A}$ and the above construction cannot be carried out. Nevertheless, one can "regularize" the interaction by introducing $V_{\eta}$, depending on a small parameter $\eta$, such that $V_{\eta} \in \mathfrak{A}$ and in any regular representation $\pi$ of the algebra $\mathfrak{A}, \pi\left(V_{\eta}\right) \rightarrow$ $G \otimes \Phi_{\pi}$, as $\eta \rightarrow 0$ (strongly on a dense domain). One can then, for $\eta>0$, carry out the above construction and finally remove $\eta$ once placed in a representation. Such a procedure is decribed in [5] - and other approaches are possible. This shows we can consider that the dynamics of the coupled system is generated by the Hamiltonian

$$
H=H_{0}+G \otimes \Phi_{\omega}(g)=H_{0}+G \otimes\left(\Phi_{\text {Fock }}(g)+\operatorname{Re} \chi_{\omega}(g)\right),
$$

acting on $\mathbb{C}^{N} \otimes \mathcal{F}\left(L^{2}\left(\mathbb{R}^{d}, \mathrm{~d} x\right)\right)$.

We take an energy conserving (non-demolition) interaction $[\mathbf{1 0}, \mathbf{1 9}]$ between the system and the reservoir, which consists in taking an operator $G$ that commutes with $H_{\mathrm{S}}$,

$$
G=\operatorname{diag}\left(g_{1}, \ldots, g_{N}\right)
$$

Such models are used to investigate "phase decoherence" of the small system.

The initial system-reservoir state is disentangled, given by a density matrix

$$
P_{0}=\rho_{\mathrm{S}} \otimes|\Omega\rangle\langle\Omega|,
$$

acting on the Hilbert space $\mathbb{C}^{N} \otimes \mathcal{F}\left(L^{2}\left(\mathbb{R}^{d}, \mathrm{~d} x\right)\right)$. Here, $\rho_{\mathrm{S}}$ is an arbitrary intial system density matrix and the reservoir is in the state $\Omega$, which represents the infinitely extended continuous mode coherent state with uniformly distributed phases. The state of the coupled system at any later time $t$ is given by

$$
P(t)=\mathrm{e}^{-\mathrm{i} t H} P_{0} \mathrm{e}^{\mathrm{i} t H} .
$$

Taking the partial trace over the reservoir Hilbert space yields the reduced system density matrix,

$$
\rho_{\mathrm{S}}(t)=\operatorname{Tr}_{\mathrm{R}} P(t) \text {. }
$$

We denote its matrix elements in the energy eigenbasis $\left\{\varphi_{j}\right\}_{j=1}^{N}$ (with $H_{\mathrm{S}} \varphi_{j}=e_{j} \varphi_{j}$ ) by

$$
\rho_{k, l}(t)=\left\langle\varphi_{k}, \rho_{\mathrm{S}}(t) \varphi_{l}\right\rangle=\operatorname{Tr} P(t)\left|\varphi_{l}\right\rangle\left\langle\varphi_{k}\right| .
$$

As $\left[H_{\mathrm{S}}, G\right]=0$ the populations (diagonal matrix elements) are time-independent. The off-diagonal ones exhibit time decay ("phase decoherence"). For the energy conserving model at hand, the matrix elements (2.24) can be evaluated exactly. The calculation yields (see Appendix D of [16])

$$
\begin{aligned}
\rho_{k, l}(t)= & \mathrm{e}^{-\mathrm{i} t\left(e_{k}-e_{l}\right)} \mathrm{e}^{-\mathrm{i} t\left(g_{k}-g_{l}\right) \operatorname{Re} \chi_{\omega}(g)} \\
& \times \mathrm{e}^{\frac{\mathrm{i}}{2}\left(g_{k}^{2}-g_{l}^{2}\right)\left\langle g, \frac{\sin (\varepsilon t)-\varepsilon t}{\varepsilon} g\right\rangle} \mathrm{e}^{-\frac{1}{2}\left(g_{k}-g_{l}\right)^{2}\left\langle g, \frac{1-\cos (\varepsilon t)}{\varepsilon^{2}} g\right\rangle} \\
& \times \rho_{k, l}(0) .
\end{aligned}
$$

The contribution on the right side of (2.25) is given by the free dynamics and by a random "renormalization" of the system energy due to the interaction with the coherent bath (coming from the term $G \otimes \operatorname{Re}_{\omega}(g) \mathbb{1}_{\mathrm{R}}$ in the Hamiltonian $(2.23)$ ). The two factors (2.26) are the same as in the case of a system coupled to a free bose 
gas in equilibrium at zero temperature. Therefore, the coherent states character of the reservoir is encoded entirely in the part $\mathrm{e}^{-\mathrm{i} t\left(g_{k}-g_{l}\right) \operatorname{Re} \chi_{\omega}(g)}$. The expectation of this oscillating factor is the characteristic function of the random variable $\operatorname{Re} \chi_{\omega}(g) \sim \mathcal{N}\left(0,\|\sqrt{\rho} g\|_{2}^{2}\right)$,

$$
\mathbb{E}\left[\mathrm{e}^{-\mathrm{i} t\left(g_{k}-g_{l}\right) \operatorname{Re} \chi_{\omega}(g)}\right]=\mathrm{e}^{-\frac{t^{2}}{2}\left(g_{k}-g_{l}\right)^{2}\|\sqrt{\rho} g\|_{2}^{2}} .
$$

This shows that the averaged (reduced system) density matrix $\mathbb{E}\left[\rho_{\mathrm{S}}(t)\right]$ acquires Gaussian time-decay of off-diagonals at all times, due to the coupling with the coherent reservoir, namely

$$
\left|\mathbb{E}\left[\rho_{k, l}(t)\right]\right|=\mathrm{e}^{-\frac{t^{2}}{2}\left(g_{k}-g_{l}\right)^{2}\|\sqrt{\rho} g\|_{2}^{2}} \mathrm{e}^{-\frac{1}{2}\left(g_{k}-g_{l}\right)^{2} \Gamma(t)}\left|\rho_{k, l}(0)\right|,
$$

with

$$
\Gamma(t)=\left\langle g, \frac{1-\cos (\varepsilon t)}{\varepsilon^{2}} g\right\rangle=2\left\langle g, \frac{\sin ^{2}(\varepsilon t / 2)}{\varepsilon^{2}} g\right\rangle .
$$

For small times, $\Gamma(t) \sim \frac{1}{2} t^{2}\|g\|_{2}^{2}$ is quadratic in time, but for large $t$, its behaviour as a function of $t$ depends on the infrared behaviour of the form factor $g,|g(k)| \sim|k|^{p}$ for $|k| \sim 0$. For instance, in $d=3$ dimensions and for $\varepsilon(k)=|k|$,

$$
\Gamma(t)=2 \int_{0}^{\infty}|k|^{2} \mathrm{~d}|k| \int_{S^{2}} \mathrm{~d} \Sigma|g(|k|, \Sigma)|^{2} \frac{\sin ^{2}(|k| t / 2)}{|k|^{2}} \sim \frac{\pi t}{2} \lim _{r \rightarrow 0_{+}} r^{2} \int_{S^{2}} \mathrm{~d} \Sigma|g(r, \Sigma)|^{2},
$$

assuming that the latter limit exists and is non-vanishing, meaning that $|g(r, \Sigma)| \sim$ $r^{-1}$ for small $r$. Note also that for $p>-1 / 2$, we have $\lim _{t \rightarrow \infty}\left\langle g, \cos (\varepsilon t) / \varepsilon^{2} g\right\rangle=0$ by the Riemann-Lebesgue lemma, so that $\lim _{t \rightarrow \infty} \Gamma(t)=\|g / \varepsilon\|_{2}^{2}$. For this infra-red behaviour of the form factor, the coupling to the (zero temperature) reservoir does not induce (complete) decoherence, but the coupling to the coherent reservoir does.

\section{References}

1. H. Araki, E. Woods: "Representations of canonical commutation relations describing a nonrelativistic infinite free Bose gas," J. Math. Phys. 4, 637-662 (1963).

2. H. Araki, W. Wyss: "Representations of canonical anticommutation relations," Helv. Phys. Acta 37, 136-159 (1964)

3. O. Bratteli, D.W. Robinson: Operator Algebras and Quantum Statistical Mechanics 1 and 2, Springer Verlag 1987

4. M. Combescure, D. Robert: Coherent States and Applications in Mathematical Physics, Springer Verlag 2012

5. J. Fröhlich, M. Merkli: "Thermal Ionization," Math. Phys. Anal. Geom. 7, no. 3, 239-287 (2004)

6. C.W. Gardiner, P. Zoller: Quantum Noise, Springer Series in Synergetics, Third Edition, 2004

7. R. J. Glauber: "The Quantum Theory of Optical Coherence", Phys. Rev. 130, 2529-2539 (1963)

8. R. J. Glauber: "Coherent and Incoherent States of the Radiation Field", Phys. Rev. 131, 2766-2788 (1963)

9. F. Grosshans, G. Van Assche, J. Wenger, R. Brouri, N. J. Cerg, Ph. Grangier: "Quantum key distribution using gaussian-modulated coherent states", Letters to Nature, Nature 421, 238-241 (2003)

10. E. Joos, H.D. Zeh, C. Kiefer, D. Giulini, J. Kupsch, I.O. Stamatescu: Decoherence and the appearence of a classical world in quantum theory. Second Edition. Springer Verlag, Berlin, 2003

11. A. Joye, M. Merkli: Representations of Canonical Commutation Relations Describing Infinite Coherent States, Commun. Math. Phys., 347, p. 421-448, (2016).

12. J. R. Klauder, B.-S. Skagerstam: Coherent states, applications in Physics and Mathematical Physics, World Scientific 1985 
13. L. Mandel, E. Wolf: Optical coherence and quantum optics, Cambridge University Press 1995

14. Ph. A. Martin, F. Rothen: Many-Body Problems and Quantum Field Theory, Springer Texts and Monographs in Physics, 2004

15. M. Merkli, G.P. Berman, R.T. Sayre, X. Wang, A.I. Nesterov: Production of Entanglement Entropy by Decoherence in Biological Systems, submitted 2017

16. M. Merkli, I.M. Sigal, G.P. Berman: "Resonance Theory of Decoherence and Thermalization," Annals of Physics 323, 373-412 (2008)

17. M. Merkli: "The Ideal Quantum Gas," in Lecture Notes in Mathematics 1880, Springer Verlag, 2006., edited by S. Attal, A. Joye, C.-A. Pillet

18. B. Øksendal, Stochastic Differential Equations, Universitext, Springer Verlag, Sixth Edition 2003

19. M.G. Palma, K.-A. Suominen, A. Ekert, "Quantum computers and dissipation," Proc. Roy. Soc. London Ser. A, 452, 567-584 (1996)

20. E. Schrödinger: "Der stetige Übergang von der Mikro- zur Makromechanik", Naturwissenschaften 14, 664-666 (1926)

21. M. Schlosshauer: Decoherence and the Quantum-to-Classical Transition, The Frontiers Collection, Springer Verlag 2007

Université Grenoble Alpes, CNRS Institut Fourier, 38000 Grenoble

E-mail address: alain.joye@univ-grenoble-alpes.fr

Department of Mathematics and Statistics, Memorial University of Newfoundland,

St. John's, NL, CANAdA, A1C 5S7

E-mail address: merkli@mun.ca 